\title{
Применение генераторов плоского поля типа GTEM-камер для радиолокационных измерений
}

\author{
А. Смирнов, д. т. н. ${ }^{1}$
}

УДК 621.317| ВАК 05.11.01

\author{
Одна из основных задач радиолокационных измерений - определение \\ эффективной площади рассеяния (ЭПР) объектов. Традиционные методы \\ измерения ЭПР рассчитаны на применение сигналов с частотами \\ выше 0,5-1 ГГц. В данной статье рассматривается метод определения \\ ЭПР с использованием GTEM-камер, позволяющий применять сигналы \\ с существенно более низкими частотами и не задействовать меры ЭПР.
}

II

змерения эффективной площади рассеяния относятся к области радиотехнических измерений и связаны с оцениванием амплитудных характеристик электромагнитного поля. Используемые для измерений радиолокационные измерительные комплексы (РИК) как открытого, так изакрытого типа фактически имитируют работу радара и применяют методы измерений замещением или сравнением с мерой. Для градуировки измерительного тракта используются входящие в состав РИК комплекты мер ЭПР, что позволяет исключить влияние многих источников погрешностей и необходимость учета многих звеньев измерительного тракта. Несмотря на длительную историю радиолокационных измерений, развитие и появление новых типов РИК, рабочий частотный диапазон реализованных измерений ограничен снизу частотой порядка 0,5-1 ГГц. Между тем ожидаемые преимущества радиолокационных обнаружителей более низкого частотного диапазона заставляют искать пути получения экспериментальных оценок ЭПР для этих частот. Однако попытки реализовать данные измерительные задачи традиционными подходами встречают проблемы как инструментального, так и методического характера. В частности:

- проблематично создать РИК традиционной конфигурации для таких больших длин волн, в основном из-за крупных полеобразующих систем;

- существенно возрастает погрешность градуировки трактов РИК из-за значительного уровня фоновых отражений в низкочастотном диапазоне;

- относительно низкая частота измерений в традиционных РИК ограничивает возможности пространственной селекции полезных сигналов при использовании широкополосных сигналов (ЛЧМ, импульсных или частотно-синтезированных);

АО «НПФ «Диполь», руководитель направления ЭМС и радиоизмерений, smirnov@dipaul.ru.
- применение мер ЭПРсбольшими номинальными значениями ЭПР (типа цилиндрических или уголковых отражателей) сдерживается возрастанием погрешности оценок действительных значений в данном частотном диапазоне, являющемся для типовых размеров мер резонансной или рэлеевской областями.

Поэтому развитие альтернативных методов измерений ЭПР и технических средств для их реализации в низкочастотном диапазоне, хотя бы для ограниченных по габаритам объектов, представляет практический интерес и является актуальным.

Одними из вероятных направлений данного развития являются следующие:

- переход от существующих методов измерений ЭПР к абсолютным методам, свободным от применения мер ЭПР и использующим принцип измерений на основе классического уравнения радиолокации либо формализованного теоретического определенияЭПр;

- анализ возможности использования и применение современных косвенных методик измерений характеристик электромагнитных полей, порождаемых объектами испытаний, реализованных в смежных областях электродинамики.

В упрощенном виде оценивание ЭПР на основе уравнения радиолокации может быть реализовано согласно выражению

$$
\sigma=\frac{P_{\text {рас }}}{P_{\text {пад }}} \frac{\left(4 \pi R^{2}\right)^{2}}{G \cdot A},
$$

где $\sigma$ - ЭПР объекта, $P_{\text {пад }}$ - входная мощность излучающей антенны; $P_{\text {рас }}$ - выходная мощность приемной антенны; $\mathrm{R}$ - расстояние до объекта измерений; G - коэффициент усиления излучающей антенны; $A$ - эффективная площадь приемной антенны.

Анализ (1) показал, что для измерений ЭПР необходим учет многих составляющих измерительного тракта. 
Реализация методики требует оценки действительных характеристик элементов тракта, а также их стабильности по времени и по динамическому диапазону измерений. В результате ожидаемое значение погрешности является достаточно высоким даже для простейшего варианта уравнения (1). Кроме того, не исчезают технические проблемы по созданию падающего и регистрации рассеянного поля, характерные для низкочастотного диапазона. Поэтому подобная методика не может быть реализована с учетом предъявляемых требований по погрешности и динамическому диапазону.

Формализованное теоретическое определение ЭПр включает в себя значения падающего и рассеянного поля и дается выражением [1]:

$$
\sigma=\lim _{R \rightarrow \infty} 4 \pi R^{2} \frac{E_{\text {рас }}^{2}}{E_{\text {пад }}^{2},}
$$

где $E_{\text {пад }}$ - напряженность падающего поля непосредственно у объекта; $E_{\text {рас }}$ - напряженность рассеянного поля на расстоянии R от объекта измерений.

Стремление Rк бесконечности необходимо для независимости величины ЭПР от расстояния. Реально же достаточно выбрать R, начиная с которого изменение напряженности $E_{\text {рас }}$ с расстоянием имеет характер обратно пропорциональной зависимости, а падающее электромагнитное поле подобно плоской волне.

Непосредственная реализация методики измерений ЭПр по (2) включала бы два измерения напряженности поля и фактически означала бы непосредственную реализацию измерений физической величины, в данном случае ЭПР, по определению. Первое измерение включало бы оценку падающего поля, второе - оценку рассеянного поля. Но данный подход не получил распространения по следующим причинам:

- трудности одновременного или синхронизованного измерения двухзначений напряженности поля в разнесенных точках пространства в широком динамическом диапазоне;

- влияние фоновых сигналов на измерение напряженности рассеянного поля

- необходимость разделения падающего и рассеянного поля;

- нестабильность трассы распространения и характеристик измерительного тракта;

- необходимость точного определения действительных значений характеристик тракта.

Между тем указанные измерительные задачи по оценке значений напряженности поля из (2) весьма похожи на задачи из области тестирования характеристик электромагнитной совместимости (ЭМС) относительно излучаемых помех. Так, задача создания падающего поля и контроля его характеристик идентична задаче тестирования устойчивости к излучаемым помехам. А измерение напряженности рассеянного поля подобно измерению эмиссии излучаемых помех. При этом, несмотря на то, что в области ЭМС данные задачи разнесены по времени или пространству, указанные проблемы генерирования и измерений характеристик электромагнитных полей, обусловленные низким частотным диапазоном, свойственны им в такой же степени. Примечательна схожесть тенденций развития радиолокационных измерительных комплексов и измерительноиспытательных систем ЭМС. В общем-то, как в радиолокационныхизмерениях, таки в области оценки характеристик ЭМС, проявились как минимум два этапа развития средств измерений. Если первый этап основывался на измерениях на открытых площадках или на открытых радиолокационных полигонах, то второй этап характеризуется переходом в закрытые полигоны, размещаемые в безэховых камерах. Можно также выделить и третий этап в каждой из областей, общим для которого является внедрение методов моделирования и методик измерений, основанных на преобразовании данных первичных измерений в финальные характеристики.

С точки зрения возможности использования методического аппарата испытаний на ЭМС применительно к радиолокационным измерениям примечательным является тот факт, что минимально требуемый частотный диапазон в испытаниях на ЭМС является сейчас востребованным для радиолокационных измерений. В частности, наиболее интенсивно измерение эмиссии излучаемых помех выполняется в диапазоне 30-1000 МГц, а тестирование устойчивости в диапазоне 80-1000 МГц. В то же время, существующие измерительные комплексы позволяют проводить измерения ЭПР в частотном диапазоне выше 0,5-1 ГГц. Поскольку измерение ЭПР, как известно, предполагает одновременное облучение полем и измерение напряженности поля, то среди инструментальных средств предпочтительными являются универсальные технические средства из области ЭМС, обеспечивающие тестирование как по эмиссии, так и по устойчивости относительно излучаемых помех.

В этой связи, целесообразно обратить внимание на полеобразующие структуры типа GTEM-камер и связанные с ними методики тестирования устойчивости и методики измерений эмиссии излучаемых помех [2]. По сути GTЕМ-камера - это полосковая структура переменного поперечного сечения с согласованной нагрузкой на одной стороне. Центральный проводник (так называемый septum) представляет собой плоскую панель, смещенную несколько в сторону от оси камеры для увеличения рабочего объема. Основными характеристиками GTEM-камер являются частотные диапазоны (полный и рабочий) и допустимые габариты объекта испытаний.

Полный частотный диапазон GTEM-камеры как полосковой структуры широк и занимает частоты практически от 0 Гц до 18-20 ГГц (по данным производителей: Teseq, ETS-Lindgren и др.). Рабочий частотный диапазон более 
узкий за счет уменьшенной верхней рабочей частоты, ограниченной допустимым КСВН или вариациями поля в рабочем объеме.

Изначально GTEM-камеры использовались как генераторы электромагнитного поля при тестировании устойчивости к излучаемым помехам. Размеры рабочего объема (габариты объекта испытаний), определяемые положением центрального проводника, составляют 1/3 от его высоты. При этом напряженность падающего поля в середине рабочего объема оценивается просто исходя из входного напряжения $\mathrm{U}_{\text {вх }}$ и высоты центрального проводника $d$ :

$$
E_{\text {пад }}=\frac{U_{\text {вх }}}{d} .
$$

В то же время замкнутый характер пространства камеры обуславливает дополнительную возможность оценки эмиссии излучаемых помех путем косвенных измерений [2]. В частности, в случае, когда максимальные габариты объектов испытаний не превышают длины волны, модель объекта в виде случайно ориентированного вибратора является адекватной. Основываясь на этом предположении, в настоящее время разработана методика измерений эмиссии излучаемых помех в диапазоне частот до 1 ГГц, нашедшая отражение в [2]. Поскольку целью измерений эмиссии излучаемых помех является оценка максимально возможной напряженности поля помех на заданном расстоянии, то знание полной излученной мощности позволяет провести подобную оценку. Согласно [2] такая оценка проводится по результатам измерений выходных напряжений GTEM-камеры при трех ортогональных положениях размещенного внутри GTEM-камеры объекта испытаний. Затем полная излученная мощность используется для оценки напряженности поля помех на выбранном расстоянии. В целом расчетные соотношения могут быть объединены в одну формулу, связывающую непосредственно выходное напряжение GTEM-камеры (среднеквадратическое значение трех компонент) и напряженность создаваемого поля помех на заданном расстоянии. Тогда итоговая формула для напряженности поля помех описывается выражением:

$$
E_{\text {изл }}=\frac{1}{R} \frac{\eta \kappa}{2 \pi e_{N}} \frac{\mathrm{U}_{\mathrm{Bdx}}}{\sqrt{z_{c}}}
$$

где $E_{\text {изл }}$ - напряженность поля помех, R - измерительное расстояние; $\eta$-волновое сопротивление свободного про-

странства, $120 \pi$ Ом; $k=\frac{2 \pi}{\lambda}-$ волновое число, $\lambda-$ длина вол-

ны; $e_{\mathrm{N}}$ - градуировочный коэффициент GTЕМ-камеры в режиме генерации поля в рабочей зоне, связывающий входную мощность $P_{\text {вх }}$ и напряженность падающего поля $E_{\text {пад' }}$ $e_{\mathrm{N}}=\frac{E_{\text {пад }}}{\sqrt{\mathrm{P}_{\mathrm{Bx}}}}=\frac{\sqrt{z_{c}}}{d}, z_{c}-$ волновое сопротивление линии, номи- нально 50 Ом, d - расстояние между проводниками в GTEM-камере в сечении рабочего объема; $U_{\text {вых }}=\sqrt{U_{x, \text { вых }}^{2}+U_{y, \text { вых }}^{2}+U_{z, \text { вых }}^{2}}-$ модуль выходного напряжения по результатам измерений выходных напряжений для трех ортогональных положений (x, $y, z)$ объекта испытаний.

Таким образом, если внутри камеры разместить объект и на вход камеры подать некоторое входное напряжение $\mathrm{U}_{\text {вх' }}$ создающее падающее поле $\mathrm{E}_{\text {пад' }}$ то возникшее выходное напряжение GTEM-камеры $U_{\text {вых }}$, являющееся результатом $\mathrm{E}_{\text {пад, }}$ позволит рассчитать напряженность поля $\mathrm{E}_{\text {изл' }}$ созданного объектом на расстоянии R. Поскольку напряжения $U_{\text {вх }}$ и $U_{\text {вых }}$ измеряются в одном сечении GTEM-камеры, то можно выразить их отношение в терминах S-параметров. При измерениях ЭПР наиболее часто измеряются ЭПР на согласованных поляризациях. При традиционном положении объекта облучающее поле является вертикально поляризованным. Следовательно, в рассеянном сигнале также интерес представляет единственная вертикальная компонента. Поэтому формально полагая, что

$$
E_{\text {рас }}=E_{\text {изл' }}
$$

и подставляя вместе с $E_{\text {пад }}$ в (2), получаем выражение для оценки ЭПР при единственном положении объекта внутри GTEM-камеры:

$$
\sigma=4 \pi\left(\frac{\eta d^{2}}{\lambda z_{c}} \frac{U_{\mathrm{Bbx}}}{U_{\mathrm{BX}}}\right)^{2}
$$

или следующее окончательное выражение для ЭПР в терминах S-параметров:

$$
\sigma=4 \pi\left(\frac{\eta d^{2}}{\lambda z_{c}}\right)^{2}\left|S_{11}\right|^{2}
$$

Полученное выражение (7) отражает принцип разработанного абсолютного метода измерений ЭПР с учетом ограничений и условий применимости [2]. Развитая на его основе методика измерений не требует использования мер ЭПР.

Для опробования методики и проверки заложенного принципа были проведены экспериментальные измерения ЭПР металлических сфер диаметрами 81 и 112 мм.

Измерительная установка представляла собой совокупность векторного анализатора цепей компании Keysight Technologies и GTEM-камеры компании Teseq (тип GTEM 250, высота проводника 25 см).

Процедура измерений не отличалась от традиционной для подобных измерений и включала в себя для каждого объекта:

- последовательные измерения частотной зависимости отраженного собственного фонового сигнала и сигнала объекта с фоном; 


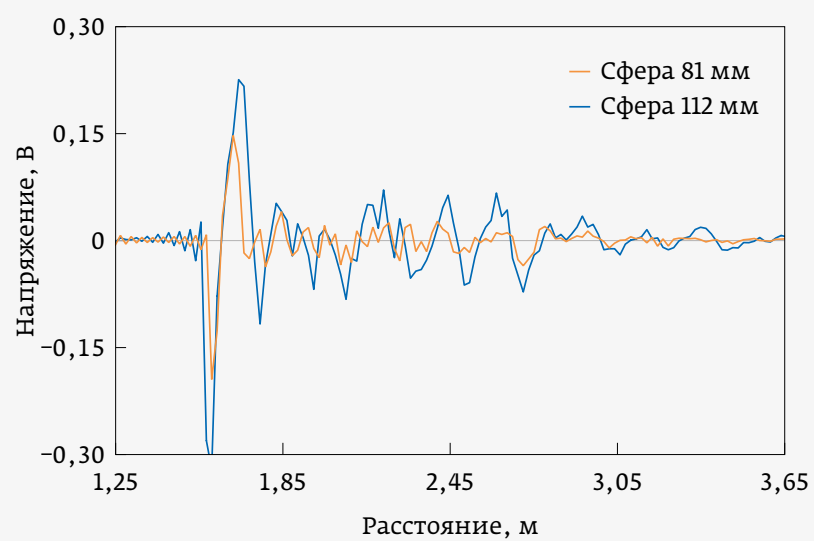

Рис. 1. Импульсные характеристики сфер, расположенных внутри СТЕМ-камеры

- последующее векторное вычитание фонового сигнала;

- преобразование частотной зависимости во временную область;

- анализ наблюдаемой импульсной характеристики и временное стробирование полезного сигнала;

- обратное преобразование в частотную область и анализ результатов частотной зависимости $S_{11}$ для «чистого" полезного сигнала.

На рис. 1 показаны измерения импульсных характеристик сфер с ярко выраженными откликами сфер и некомпенсированным фоновым сигналом, обусловленным затенением объектом части задней стенки GTEM-камеры.

На рис. 2 показаны завершающие результаты измерений ЭПР металлических сфер в широкой полосе частот.

Наблюдается хорошее совпадение с теоретическими результатами в диапазоне до 1-1,5 ГГц, что соответствует ограничениям [1]. При этом близость к теории более характерна для сферы меньшего диаметра, что опять же соответствует [1] в части соотношения габаритов объекта и высоты центрального проводника (25 см для GTEM 250). Более того, для сферы меньшего диаметра характерно хорошее совпадение в диапазоне частот до 2 ГГц, что выходит за рамки стандартизованного частотного диапазона измерений.

$$
\because ;
$$

В целом на основе проведенных исследований можно констатировать следующее:

- предложенный абсолютный метод позволяет измерять ЭПр без применения мер ЭПр;

- метод и связанная с ним методика позволяют проводить измерения ЭПр простейших тел в диапазоне частот до 1 гГц и несколько выше с приемлемой погрешностью;
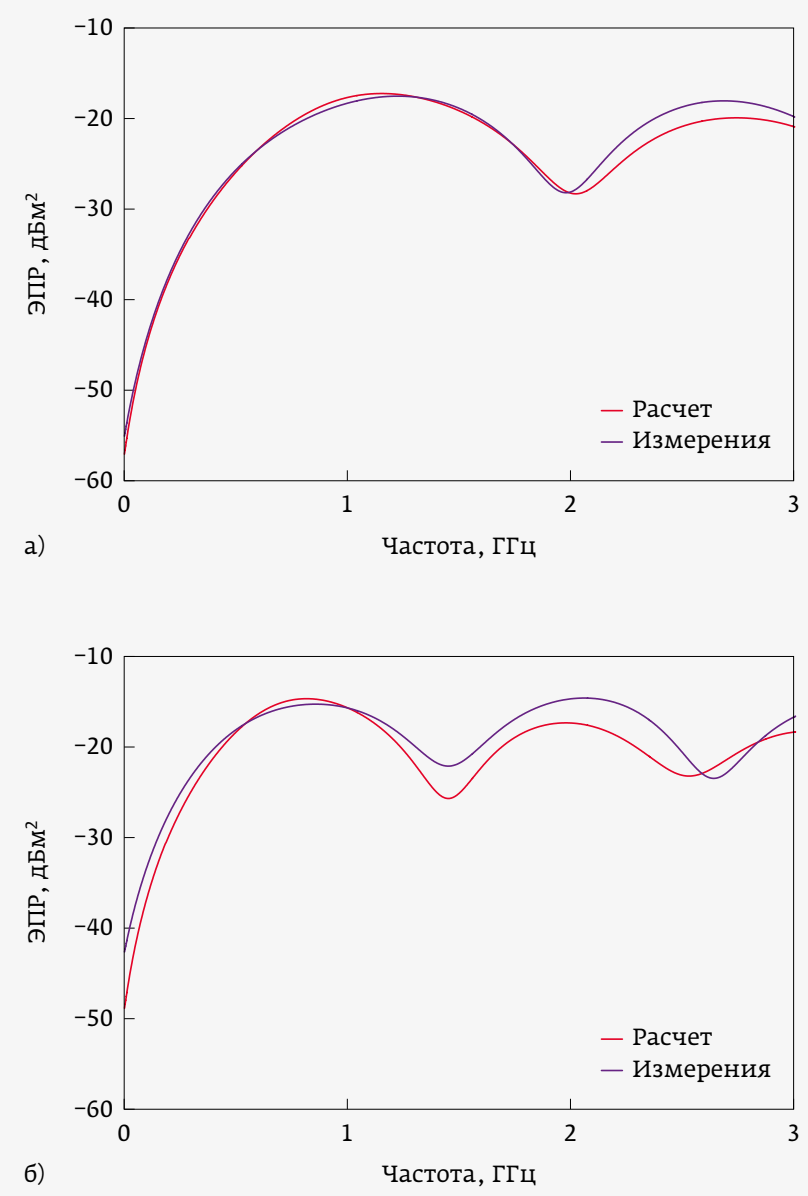

Рис. 2. Сравнительные результаты измерений и расчетов ЭПР: а - сфера 81 мм; б - сфера 112 мм

- в перспективе с помощью разработанного метода предполагается получать экспериментальные данные о действительных значениях мер эПР в рэлеевской и резонансной областях для тел простейших форм непрерывно по частотному диапазону. Возможность получения точных результатов измерений ЭПР калибровочных отражателей позволит получить нормированные данные об эПР в качестве стандартных справочных данных для частотного диапазона рэлеевской и резонансной областей.

\section{ЛИТЕРАТУРА}

1. Майзельс Е.Н., Торгованов В.А. Измерение характеристик рассеяния радиолокационных целей / Под ред. М. А. Колосова. - М.: Сов. радио, 1972. 232 С.

2. IЕC 61000-4-20 (2010). Электромагнитная совместимость. Часть 4-20. Методики испытаний и измерений. Испытание на эмиссию и невосприимчивость в поперечных электромагнитных волноводах. 